\title{
On compactness in the Trudinger-Moser inequality
}

\author{
ADIMURTHI AND CYRIL TINTAREV
}

\begin{abstract}
We show that the Moser functional $J(u)=\int_{\Omega}\left(e^{4 \pi u^{2}}-1\right) \mathrm{d} x$ on the set $\mathcal{B}=\left\{u \in H_{0}^{1}(\Omega):\|\nabla u\|_{2} \leq 1\right\}$, where $\Omega \subset \mathbb{R}^{2}$ is a bounded domain, fails to be weakly continuous only in the following exceptional case. Define $g_{s} w(r)=s^{-\frac{1}{2}} w\left(r^{s}\right)$ for $s>0$. If $u_{k} \rightarrow u$ in $\mathcal{B}$ while $\liminf J\left(u_{k}\right)>J(u)$, then, with some $s_{k} \rightarrow 0$,

$$
u_{k}=g_{s_{k}}\left[(2 \pi)^{-\frac{1}{2}} \min \left\{1, \log \frac{1}{|x|}\right\}\right],
$$

up to translations and up to a remainder vanishing in the Sobolev norm. In other words, the weak continuity fails only on translations of concentrating Moser functions. The proof is based on a profile decomposition similar to that of Solimini [16], but with different concentration operators, pertinent to the two-dimensional case.
\end{abstract}

Mathematics Subject Classification (2010): 35J20 (primary); 35J35, 35J60, 46E35, 47J30, 58E05 (secondary).

\section{Introduction}

The purpose of this paper is to study weak continuity properties in the TrudingerMoser inequality at the same level of detail as the better-understood weak continuity properties of the critical nonlinearity in higher dimensions. We draw comparisons between the Sobolev inequality that defines the continuous imbedding $\mathcal{D}^{1, p}\left(\mathbb{R}^{N}\right) \hookrightarrow$ $L^{p^{*}}$, with $p^{*}=\frac{p N}{N-p}$, when $N>p$, and the Trudinger-Moser inequality (see Yudovich [22], Peetre [14], Pohozhaev [15], Trudinger [21] and Moser [13]):

$$
\sup _{\mathcal{B}} \int_{\Omega} e^{\alpha_{N}|u|^{N^{\prime}}} \mathrm{d} x<\infty, \quad \mathcal{B}=\left\{u \in W_{0}^{1, N}(\Omega):\|\nabla u\|_{N} \leq 1\right\},
$$

where $\Omega \subset \mathbb{R}^{N}$ is a bounded domain, $N^{\prime}=\frac{N}{N-1}$, the constant $\alpha_{N}=N \omega_{N-1}^{1 /(N-1)}$ is the optimal constant (due to Moser [13]), and $\omega_{N-1}$ is the area of the unit $(N-1)$ -

Research done in part while the second author was visiting the TIFR Centre for Applicable Mathematics in Bangalore and the Chinese University of Hong Kong.

Received November 4, 2011; accepted in revised form June 6, 2012. 
dimensional sphere. Using the notation $\|u\|_{q}$ for the $L^{q}$-norm of $u$, we fix the norm of $W_{0}^{1, N}$ as $\|\nabla u\|_{N}$. The ball in $\mathbb{R}^{N}$ of radius $R$ centered at $y$ will be denoted by $B_{R}(y)$, abbreviated to $B_{R}$ when $y=0$, and to $B$ if $y=0$ and $R=1$. We will refer to the functional

$$
J(u) \stackrel{\mathrm{d} e f}{=} \int_{\Omega}\left(e^{\alpha_{N}|u|^{N^{\prime}}}-1\right) \mathrm{d} x
$$

as the Moser functional. Analogues of the Trudinger-Moser inequality have been established for more general Sobolev spaces by Adams ([1], the case of higher derivatives), and by Fusco, Lions and Sbordone ([8], a weighted version of the Trudinger-Moser inequality that allows nonlinearities of very high growth). The imbeddings $\mathcal{D}^{1, p}\left(\mathbb{R}^{N}\right) \hookrightarrow L^{p^{*}}$, with $p<N$, and $W_{0}^{1, N}(B) \hookrightarrow \exp L^{N^{\prime}}$, defined, respectively, by the Sobolev and the Trudinger-Moser inequalities, are optimal when the target space in the class of Orlicz spaces. Further refinement of these imbeddings is possible, in the larger class of rearrangement-invariant spaces, where the correspondent Orlicz spaces can be identified on the scales of Lorentz, respectively Lorentz-Zygmund, spaces as $L^{p^{*}}=L^{p^{*}, p^{*}}$, respectively $\exp L^{N^{\prime}}=$ $L^{\infty, \infty ;-1 / N}$. For further details we address the reader to Appendix A.

It is well known that the critical Sobolev nonlinearity $\int_{\mathbb{R}^{N}}|u|^{p^{*}} \mathrm{~d} x$ lacks weak continuity in $\mathcal{D}^{1, p}\left(\mathbb{R}^{N}\right)$ at any point $u$ (consider any sequence of the form $u_{k}(x)=$ $u(x)+k^{\frac{N-p}{p}} w(k x)$, with $w \neq 0$, and $k \rightarrow \infty$, and apply the Brezis-Lieb lemma). In contrast to that, according to the result of Lions, Theorem I.6 in ( [12], Moser functional on $\mathcal{B}$ is weakly continuous at any point except zero, and also weakly continuous on every sequence in $\mathcal{B}$ that converges weakly to zero, unless $\left\|\nabla u_{k}\right\|_{N} \rightarrow 1$ and $u_{k}$ has exactly one point of concentration).

Restricted to radially symmetric functions, the result of Lions can be further refined using calculations from the paper [13] of Moser, that allow to infer that the Moser functional on $\mathcal{B}$ lacks weak continuity only (up to a remainder vanishing in $W_{0}^{1, N}$ ) on a sequence of Moser functions (1.3) concentrating at the origin. We reproduce these calculations in Appendix B.

This indicates that, even without the assumption of radial symmetry, the class of sequences on which the Moser functional fails to be weakly continuous may be characterized not just by the mere property of concentration at one point, but by a specific asymptotic behavior. The main result of this paper, proved here for the case $N=p=2$, is that this class consists of the same sequences as in the radial case, but subject to arbitrary translations. This is consistent with the observation made here that the concentrating sequences that do not vanish in the $\exp L^{2}$-norm are always asymptotically radial, in contrast with the case $N>2$. By a concentrating sequence we mean here a sequence bounded in the corresponding Sobolev space, convergent almost everywhere to zero, but not vanishing in the $L^{p^{*}}$-norm for $N>p$, respectively in $\exp L^{N^{\prime}}$-norm for $N=p$. In the case $N>p$, any (generally nonradial) function $w$ can occur as a concentration profile, since the sequence $u_{k}(x)=k^{\frac{N-2}{2}} w(k x)$ will be a concentrating sequence, whose normalized deflations by the scale factor $k^{-1}$, that is, $k^{-\frac{N-2}{2}} u_{k}\left(k^{-1} x\right)$, equal $w$. In the case 
$N=p=2$ the analogous sequence $u_{k}(x)=w(k x)$, vanishes in $\exp L^{2}$, i.e. it is not concentrating. The relevant counterpart of the rescaling deflations, presented in this paper for $N=2$, forms sequences with discrete rotational symmetries whose rank goes to infinity, forming radial concentration profiles in the limit.

In order to define a concentration that describes, for sequences bounded in the $H_{0}^{1}$-norm, the defect of convergence in the $\exp L^{2}$-norm, we prove a suitable profile decomposition, similar to the decomposition in [4] for the radial case. Our starting point (we do not survey here a vast earlier literature where profile decompositions are established under substantial additional assumptions, typically, for critical sequences for elliptic variational problems, mentioning only the pioneering work of Struwe [18]) is the profile decomposition due to Solimini [16] (a similar decomposition was independently proved by Gerard [9] and extended to more general spaces by Jaffard [10], as well as by one of the authors of this paper) expresses a subsequence of an arbitrary bounded sequence in the Sobolev space $\mathcal{D}^{1, p}\left(\mathbb{R}^{N}\right)$, with $N>p$, as an asymptotic sum whose terms have the form $t_{k}^{\frac{N-p}{p}} w\left(t_{k} x\right)$, with a remainder vanishing in $L^{p^{*}}$. In the paper [19] (elaborated in [20]) existence of a profile decomposition was proved for a general Hilbert space equipped with a group (subject to some general conditions) of unitary operators. This in turn gave rise to the notion of cocompact imbedding of Banach spaces $X \hookrightarrow Y$, which, roughly speaking, amounts to the vanishing in the norm of $Y$ of the remainder of the profile decomposition in $X$. The functional-analytic profile decomposition prompted us to define operators that could play the role of Solimini's "rescalings", with the remainder in the profile decomposition vanishing in $\exp L^{N^{\prime}}$. In [4], dealing with the subspace of radial functions of $W_{0}^{1, N}(B)$, pertinent rescalings are given by (B.4). In this paper we have adopted an extension of the unitary operators (B.4) for the case $N=2$ to isometires $(2.15)$ on the whole space $W_{0}^{1, N}(B)$. These isometries are no longer bijective. Furthermore, they are defined only for integer values of the parameter, and, while the operators (B.4) form a group, the set of operators (2.15) is only a semigroup. On the other hand, it is exactly the absence of bijectivity (in fact, of surjectivity, since isometries are always injective) that is ultimately responsible for radiality of concentration profiles.

The results of the paper are as follows. In order to establish the structure of the exceptional sequences for the Moser functional, we employ a straightforward adaptation, Theorem 2.2, of the functional-analytic profile decomposition theorem from [20]. Theorem 2.5 is an application of Theorem 2.2 to the Sobolev space $H_{0}^{1}(B)$ equipped with the semigroup (2.15). In Theorem 2.6 we verify that the remainder of the profile decomposition vanishes in the $\exp L^{2}$-norm (which is an equivalent quasinorm of $L^{\infty, \infty ;-1 / 2}$, or in other words, that the imbedding $H_{0}^{1}(B) \hookrightarrow L^{\infty, \infty ;-1 / 2}$ is cocompact. Combining this and the optimal imbedding $H_{0}^{1}(B) \hookrightarrow L^{\infty, 2 ;-1}$, one gets by the Hölder inequality that the imbedding $H_{0}^{1}(B) \hookrightarrow L^{\infty, q ;-1 / q-1 / 2}$ is cocompact for any $q>2$. By analogy with Solimini's counterexample on [16, page 333], we also show that the optimal imbedding $H_{0}^{1}(B) \hookrightarrow L^{\infty, 2 ;-1}$ is not cocompact. The main result of the paper is as follows: 
Theorem 1.1. Let $\Omega \subset \mathbb{R}^{2}$ be a bounded domain, and let $J$ be the Moser functional (1.2). If $u_{k} \in H_{0}^{1}(\Omega)$ is such that $\left\|\nabla u_{k}\right\|_{2} \leq 1, u_{k} \rightarrow u$, and $\lim \inf J\left(u_{k}\right)>J(u)$, then there is a sequence $\zeta_{k} \in \bar{\Omega}$ and a sequence $s_{k} \in(0,1)$ such that $u_{k}-m_{s_{k}}(\cdot-$ $\left.\zeta_{k}\right) \rightarrow 0$ in the $H^{1}$-norm, where

$$
m_{s}(r) \stackrel{\mathrm{d} e f}{=}\left(\omega_{N-1}\right)^{-\frac{1}{N}} \log (1 / s)^{\frac{1}{N^{\prime}}} \min \left\{\frac{\log (1 / r)}{\log (1 / s)}, 1\right\}, \quad r, s \in(0,1) .
$$

The functions (1.3) were used by Moser in [13] to prove optimality of the constant in (1.1), and they are usually called Moser functions.

Profile decompositions, Theorem 2.2 and Theorem 2.5 are proved in Section 2. The proofs of Theorem 2.6 and of Theorem 1.1 are given in Section 3. Appendix A provides some background material on imbeddings of Sobolev spaces into Lorentz and Lorentz-Zygmund spaces, and Appendix B summarizes properties of the Moser functional in the radial case.

ACKnowledgements. One of the authors (T.) thanks Michael Cwikel, Luboš Pick and Yevgeniy Pustylnik for discussions in connection to Appendix A, and Sergio Solimini for enlightening comments about profile decompositions.

\section{Profile decomposition in $H_{0}^{1}$}

We give below a definition of isometric dislocations, extending the definition of dislocation operators from [20] to the case of non-surjective isometries.

Definition 2.1. Let $H_{1}$ be a separable infinite-dimensional Hilbert space and let $H_{0}$ be a closed subspace of that. A set $D$ of isometric linear operators from $H_{0}$ to $H_{1}$ is a set of isometric dislocations if, whenever $u_{k} \in H$ and $g_{k}, h_{k} \in D$,

$$
g_{k} u_{k} \rightarrow 0, \quad h_{k} g_{k}^{*} \nrightarrow 0 \Rightarrow \exists\left\{k_{j}\right\} \subset \mathbb{N}, \quad \text { s.t. } \quad h_{k_{j}} u_{k_{j}} \rightarrow 0 .
$$

One says that a sequence $u_{k}$ is $D$-weakly convergent to zero if for every sequence $g_{k} \in D$ one has $g_{k} u_{k} \rightarrow 0$.

Note that we deviate in this section from the notation in [20], by interchanging the operator set $D$ and the set of adjoints $D^{*}=\left\{g^{*}: g \in D\right\}$. This interchange is important for coherence with the applications in this paper, while it is of no significance for the applications studied in [20], or for the most typical applications elsewhere, when $D$ is a group of unitary operators and therefore $D^{*}=D$.

Theorem 2.2. Let $H_{1}$ be a separable infinite-dimensional Hilbert space with a closed subspace $H_{0}$ and a set of isometric dislocations $D: H_{0} \rightarrow H_{1}$. If $u_{k} \in H_{0}$ is a bounded sequence and $u_{k} \rightarrow 0$ then there exists a set $\mathbb{N}_{0} \subset \mathbb{N}, w^{(n)} \in H$, 
$g_{k}^{(n)} \in D, g_{k}^{(1)}=i d$, with $k \in \mathbb{N}$ and $n \in \mathbb{N}_{0}$, such that, for a renumbered subsequence,

$$
\begin{aligned}
& w^{(n)}=w \text {-lim } g_{k}^{(n)} u_{k}, \\
& g_{k}^{(n)} g_{k}^{(m)^{*}} \rightarrow 0 \text { for } n \neq m, \\
& \sum_{n \in \mathbb{N}_{0}}\left\|w^{(n)}\right\|^{2} \leq \lim \sup \left\|u_{k}\right\|^{2}, \\
& u_{k}-\sum_{n \in \mathbb{N}_{0}} g_{k}^{(n)^{*}} w^{(n)} \stackrel{D}{\rightarrow} 0,
\end{aligned}
$$

where the series $\sum_{n \in \mathbb{N}_{0}} g_{k}^{(n)^{*}} w^{(n)}$ converges uniformly in $k$.

Proof. The proof is an elementary modification of the proof of Theorem 3.1 from [20] and we give it in an abbreviated form.

1. One shows first that (2.4) follows from (2.2) and (2.3). The proof of this step is analogous to that in [20] and can be omitted.

2. Observe that if $u_{k} \stackrel{D}{\rightarrow} 0$, the theorem is verified with $\mathbb{N}_{0}=\emptyset$. If not so, consider the expressions of the form

$$
w^{(1)}=: \mathrm{w}-\lim g_{k}^{(1)} u_{k} .
$$

Since we assume that $u_{k}$ does not converge $D$-weakly to zero, there exists necessarily a renumbered sequence $g_{k}^{(1)}$ that yields a non-zero limit in (2.6).

Let $v_{k}^{(1)}=u_{k}-g_{k}^{(1) *} w^{(1)}$, and observe that, by (2.6),

$$
g_{k}^{(1)} v_{k}^{(1)}=g_{k}^{(1)} u_{k}-w^{(1)} \rightarrow 0 .
$$

If $v_{k}^{(1)} \stackrel{D}{\rightarrow} 0$, the theorem is verified with $\mathbb{N}_{0}=\{1\}$. If not - we repeat the argument above - there exist, necessarily, a sequence $g_{k}^{(2)} \in D$ and a $w^{(2)} \neq 0$ such that, on a renumbered subsequence, $g_{k}^{(2)} v_{k}^{(1)} \rightarrow w^{(2)}$. Let us set $v_{k}^{(2)}=v_{k}^{(1)}-g_{k}^{(2) *} w^{(2)}$. Then we will have an obvious analog of (2.7):

$$
g_{k}^{(2)} v_{k}^{(2)}=g_{k}^{(2)} v_{k}^{(1)}-w^{(2)} \rightarrow 0 .
$$

If we assume that $g_{k}^{(1)} g_{k}^{(2)^{*}} \nrightarrow 0$, then by (2.1), (2.8), $g_{k}^{(1)}\left(v_{k}^{(1)}-g_{k}^{(2)^{*}} w^{(2)}\right) \rightarrow 0$, which, due to (2.7), yields

$$
g_{k}^{(1)} g_{k}^{(2) *} w^{(2)} \rightarrow 0 .
$$

We now use (2.1) again to replace in (2.9) $g_{k}^{(1)}$ with $g_{k}^{(2)}$, which results in

$$
w^{(2)} \rightarrow 0,
$$


which cannot be true since we assumed $w^{(2)} \neq 0$. This contradiction implies that $g_{k}^{(1)} g_{k}^{(2)^{*}} \rightarrow 0$. Since for bounded sequences of operators the condition $A_{k} \rightarrow 0$ implies $A_{k}^{*} \rightarrow 0$, we also have $g_{k}^{(2)} g_{k}^{(1)^{*}} \rightarrow 0$. Recursively we define

$$
v_{k}^{(n)}:=v_{k}^{(n-1)}-g_{k}^{(n)^{*}} w^{(n)}=u_{k}-g_{k}^{(1)^{*}} w^{(1)}-\cdots-g_{k}^{(n)^{*}} w^{(n)},
$$

where

$$
w^{(n)}=\mathrm{w}-\lim g_{k}^{(n)} v_{k}^{(n-1)},
$$

calculated on a successively renumbered subsequence. We subordinate the choice of $g_{k}^{(n)}$ and thus the extraction of this subsequence for every given $n$ to the following requirements. For every $n \in \mathbb{N}$ we set

$$
W_{n}=\left\{w \in H_{1} \backslash\{0\}: \exists g_{j} \in D,\left\{k_{j}\right\} \subset \mathbb{N} \text { such that } g_{j} v_{k_{j}}^{(n)} \rightarrow w\right\},
$$

and $t_{n}=\sup _{w \in W_{n}}\|w\|$. If for some $n$, we have $t_{n}=0$, the theorem is proved. Otherwise, like in [20] we choose $w^{(n+1)} \in W_{n}$ such that

$$
\left\|w^{(n+1)}\right\| \geq \frac{1}{2} t_{n}
$$

and the sequence $g_{k}^{(n+1)}$ is chosen so that, on a subsequence that we renumber,

$$
g_{k}^{(n+1)} v_{k}^{(n)} \rightarrow w^{(n+1)} .
$$

An argument analogous to that used above for $n=1,2$ shows that

$$
g_{k}^{(p)} g_{k}^{(q)^{*}} \rightarrow 0 \text { whenever } p \neq q, p, q \leq n .
$$

This allows to immediately deduce (2.2) from (2.13).

3. Similarly to [20] one derives that $t_{n} \rightarrow 0$, from which it then follows the asymptotics (2.5). The convergence of the series (2.5), like in [20], is a modification of the Plancherel formula that requires to extract a sufficiently rarefied subsequence of $u_{k}$ to assure sufficient approximation of orthogonality by the asymptotically orthogonal terms $g_{k}^{(n)^{*}} w^{(n)}$.

From now on we assume, without loss of generality that $\Omega \subset B_{\frac{1}{2}}$. This restriction is not substantial and can be removed by linear rescaling, since, if we denote the Moser function subordinated to an annulus $t<r<R$ as $m_{t}^{(R)}$, an easy computation shows that, for any $R>0$,

$$
\lim _{t \rightarrow 0}\left\|\nabla\left(m_{t}^{(R)}-m_{t}\right)\right\|_{2} \rightarrow 0
$$


Let us now specify $H_{1}, H_{0}$ and $D$ as follows: $H_{1}=H_{0}^{1}(B), H_{0}=H_{0}^{1}(\Omega)$, and

$$
D=\left\{g_{j, \zeta} u(z)=j^{-\frac{1}{2}} u\left(\zeta+z^{j}\right), \zeta \in \bar{\Omega}, j \in \mathbb{N}\right\} .
$$

Here and in what follows, the expression $z^{j}$ stands for the power of the complex number representing a point in $z \in \mathbb{R}^{2}$, and translations of functions in $H_{0}^{1}(\Omega)$ are understood, using extension by zero, as elements of $H_{0}^{1}(B)$.

We have the following obvious property of the asymptotic profiles: (2.2).

Remark 2.3. If the sequences $u_{k} \in H_{0}^{1}(\Omega), j_{k} \in \mathbb{N}$, and $\zeta_{k} \in \Omega$ are such that $g_{j_{k}, \zeta_{k}} u_{k} \rightarrow w, j_{k} \rightarrow \infty$ and $z_{k} \rightarrow z_{0}$, then $w$ is radially symmetric.

There is also an obvious analytic form of (2.3):

Lemma 2.4. Let $D$ be the set of operators as above. Two sequences, $\left\{g_{j_{k}^{(1)}, z_{k}^{(1)}}\right\}_{k} \subset$ $D$ and $\left\{g_{j_{k}^{(2)}, z_{k}^{(2)}}\right\}_{k} \subset D$, with $j_{k}^{(1)} \rightarrow \infty$ and $j_{k}^{(2)} \rightarrow \infty$, satisfy

$$
g_{j_{k}^{(2)}, z_{k}^{(2)}} g_{j_{k}^{(1)}, z_{k}^{(1)}}^{*} \rightarrow 0
$$

if and only if

$$
\inf _{k}\left|z_{k}^{(2)}-z_{k}^{(1)}\right|>0 \text { or }\left|\log j_{k}^{(2)}-\log j_{k}^{(1)}\right| \rightarrow \infty .
$$

This allows to express Theorem 2.2 for our particular choice of $H_{1}, H_{0}$ and $D$ as follows:

Theorem 2.5. Let $\Omega \subset B_{\frac{1}{2}} \subset \mathbb{R}^{2}$ and let $u_{k} \rightarrow 0$ be a sequence in $H_{0}^{1}(\Omega)$. There exist $j_{k}^{(n)} \in \mathbb{N}$, with $\lim _{k \rightarrow \infty} j_{k}^{(n)}=\infty$, and $z_{k}^{(n)} \in \bar{\Omega}$, with $\lim _{k \rightarrow \infty} z_{k}^{(n)}=z_{n} \in \bar{\Omega}$, $k \in \mathbb{N}, n \in \mathbb{N}$, such that, for a renumbered subsequence,

$$
\begin{aligned}
& w^{(n)}(|z|)=w-\lim \left(j_{k}^{(n)}\right)^{-1 / 2} u_{k}\left(z_{k}^{(n)}+z^{j_{k}^{(n)}}\right), \\
& z_{m} \neq z_{n} \text { or }\left|\log j_{k}^{(m)}-\log j_{k}^{(n)}\right| \rightarrow \infty \text { whenever } n \neq m, \\
& \sum_{n \in \mathbb{N}} \int_{B}\left|\nabla w^{(n)}\right|^{2} \mathrm{~d} x \leq \lim \sup \int_{\Omega}\left|\nabla u_{k}\right|^{2} \mathrm{~d} x, \\
& u_{k}-\sum_{n \in \mathbb{N}} j_{k}^{(n)}{ }^{1 / 2} w^{(n)}\left(\left|z-z_{n}\right|^{1 / j_{k}^{(n)}}\right) \stackrel{D}{\rightarrow} 0,
\end{aligned}
$$

and the series $\sum_{n \in \mathbb{N}} j_{k}^{(n)^{1 / 2}} w^{(n)}\left(\left|z-z_{n}\right|^{1 / j_{k}^{(n)}}\right)$ converges in $H_{0}^{1}(B)$ uniformly in $k$.

We also note that for any radially symmetric function $w \in H_{0}^{1}(B)$ ), the sequence $g_{j, \zeta}{ }^{*} w=j^{\frac{1}{2}} w\left(|z-\zeta|^{j}\right)$ is dependent on $\zeta$ continuously in $H_{0}^{1}(B)$ and uniformly in $j \in \mathbb{N}$, so in the asymptotic expansion (2.20) we could replace $g_{j_{k}^{(n)}, z_{k}^{(n)}}^{*} w^{(n)}$ by $g_{j_{k}^{(n)}, z_{n}}^{*} w^{(n)}$. 
We complement this profile decomposition by the next statement, which identifies the convergence of the remainder in (2.20) as convergence in the Banach space $\exp L^{2}$ :

Theorem 2.6. Let $\Omega \subset B_{\frac{1}{2}}$, and let $D$ be the set (2.15). If a sequence $u_{k} \in H_{0}^{1}(\Omega)$ is D-weakly convergent to zero, then $u_{k} \rightarrow 0$ in $\exp L^{2}$. In particular, for any $\lambda>0$, we have $\int_{\Omega}\left(e^{\lambda u_{k}^{2}}-1\right) \mathrm{d} x \rightarrow 0$.

Note that the restriction $\Omega \subset B_{\frac{1}{2}}$ is not substantial and this statement can be restated for any bounded domain by linear rescaling.

Before we prove the theorem, we state a corollary and a counterexample to it in the limit case $q=2$.

Corollary 2.7. Let $\Omega \subset B_{\frac{1}{2}}$. If a sequence $u_{k} \in H_{0}^{1}(\Omega)$ is D-weakly convergent to zero, then, for every $q \in(2, \infty]$, it converges to zero in the Lorentz-Zygmund space $L^{\infty, q ;-1 / q-1 / 2}$.

This easily follows from the embedding $H_{0}^{1}(B) \hookrightarrow L^{\infty, 2 ;-1}$ and the interpolation by Hölder inequality between $L^{\infty, 2 ;-1}$ and $L^{\infty, \infty ;-1 / 2}=\exp L^{2}$.

Remark 2.8. By analogy with the counterexample given by Solimini [16] that the remainder in his profile decomposition does not necessarily converge in the sense of $L^{p^{*}, p}$, we can show that Corollary 2.7 does not extend to the endpoint case $q=2$. Our construction of the sequence is analogous to Solimini's.

Let $v \in C_{0}^{\infty}\left(\left(e^{-3}, e^{-2}\right)\right), v \neq 0$, let $w(x)=v(|x|)$ and let

$$
w_{k}=k^{-1 / 2} \sum_{j=1}^{k} g_{2^{j}, 0} w, k \in \mathbb{N} \text {. }
$$

Let us show that, for arbitrary sequences $j_{k} \rightarrow \infty$ and $\zeta_{k} \in \Omega$, one has $g_{j_{k}, \zeta_{k}} w_{k} \rightarrow 0$.

By the standard density argument, it suffices to prove that $\int g_{j_{k}, \zeta_{k}} w_{k} \psi \rightarrow 0$ for each $\psi \in L^{2}(B)$. Indeed, since the supports for the individual terms in the sum defining $w_{k}$ remain disjoint under the action of $g_{j_{k}, \zeta_{k}}$,

$$
\left(\int g_{j_{k}, \zeta_{k}} w_{k} \psi\right)^{2} \leq\left\|g_{j_{k}, \zeta_{k}} w_{k}\right\|_{2}\|\psi\|_{2},
$$

and an elementary computation shows that

$$
\left\|g_{j_{k}, \zeta_{k}} w_{k}\right\|_{2} \leq\left\|w_{k}\right\|_{2}^{2} \leq k^{-1}\|w\|_{2}^{2} \rightarrow 0
$$

Observe now that the terms in the sum in (2.21) have disjoint supports, which implies that $\left\|\nabla w_{k}\right\|_{2}=\|\nabla w\|_{2}$. Therefore we have a bounded sequence in $H_{0}^{1}(B)$ that converges $D$-weakly to zero. However, an analogous calculation also gives that

$$
\int \frac{w_{k}^{2}}{r^{2}\left(\log \frac{1}{r}\right)^{2}}=\int \frac{w^{2}}{r^{2}\left(\log \frac{1}{r}\right)^{2}} .
$$


Note that $\frac{1}{r^{2}\left(\log \frac{1}{r}\right)^{2}}$ is a decreasing function on the support of $w_{k}$, which implies that

$$
\int \frac{w^{2}}{r^{2}\left(\log \frac{1}{r}\right)^{2}} \leq \int \frac{w_{k}^{\star 2}}{r^{2}\left(\log \frac{1}{r}\right)^{2}} \leq C\left\|w_{k}\right\|_{L^{\infty, 2 ;-1 / 2}}^{2},
$$

and thus $w_{k}$ does not converge to zero in $L^{\infty, 2 ;-1 / 2}$.

\section{Proofs of Theorem 2.6 and Theorem 1.1}

The proof of Theorem 2.6 is based on the following five lemmas. None of the lemmas, except, possibly, Lemma 3.5, is a new result, but we have included them for the sake of completeness of the presentation. We recall that the expression $z^{j}$, $j \in \mathbb{N}$, refers to a power of the complex number $z$ representing a point in $\mathbb{R}^{2}$, the set of operators $D$ is defined by (2.15), and $D$-weak convergence is defined in Definition 2.1.

We start with the following elementary statement:

Lemma 3.1. Let $\bar{\Omega} \subset$ B. If $u_{k} \in H_{0}^{1}(\Omega)$ and $u_{k} \stackrel{D}{\rightarrow} 0$ then $g_{j_{k}, \zeta_{k}} u_{k} \rightarrow 0$ whenever $j_{k} \in \mathbb{N}, \zeta_{k} \in B$.

Proof. For $\zeta_{k} \in \bar{\Omega}$ the assertion follows directly from the definition of $D$-weak convergence. For $\zeta_{k} \in B \backslash \bar{\Omega}$, note that $u_{k} \stackrel{D}{\rightarrow} 0$ implies $u_{k} \rightarrow 0$ and that operators $g_{j_{k}, \zeta_{k}}$ map any sequence $u_{k} \rightarrow 0$, such that $\inf _{k} \operatorname{dist}\left(\zeta_{k}, \operatorname{supp} u_{k}\right)>0$ to a sequence that weakly converges to zero. Finally, the case $\operatorname{dist}\left(\zeta_{k}, \operatorname{supp} u_{k}\right) \rightarrow 0$ can be easily reduced by a continuity argument to the case $\zeta_{k} \in \bar{\Omega}$.

In what follows, the two-dimensional Lebesgue measure will be denoted by $\mathrm{d} x \mathrm{~d} y$ when the integration variable is $z$, and by $\mathrm{d} \xi \mathrm{d} \eta$ when the integration variable is called $\zeta$. Let us introduce the averaging operator

$$
A_{r} u(z)={ }_{B_{r}(z)} u(\zeta) \mathrm{d} \xi \mathrm{d} \eta=\frac{1}{\left|B_{r}(z)\right|} \int_{B_{r}(z)} u(\zeta) \mathrm{d} \xi \mathrm{d} \eta .
$$

Lemma 3.2. Let $u_{k} \in H_{0}^{1}(\Omega), \bar{\Omega} \subset B$. If $u_{k} \stackrel{D}{\rightarrow} 0$ and $r_{k} \downarrow 0$, then $A_{r_{k}} u_{k} \stackrel{D}{\rightarrow} 0$.

Proof. Without loss of generality, assume that $u_{k} \geq 0$. It suffices to verify that for each nonnegative $\varphi \in C_{0}^{1}(B)$ and for each sequence $j_{k} \in \mathbb{N}, \zeta_{k} \in \bar{\Omega}$,

$$
\int_{B} \varphi(z) g_{j_{k}, \zeta_{k}} A_{r_{k}} u_{k}(z) \mathrm{d} x \mathrm{~d} y \rightarrow 0 .
$$


Then we have

$$
\begin{aligned}
& \int_{B} \varphi(z) g_{j_{k}, \zeta_{k}}\left(f_{B_{r_{k}}} u_{k}(z+\zeta) \mathrm{d} \xi \mathrm{d} \eta\right) \mathrm{d} x \mathrm{~d} y \\
& =\int_{B} j_{k}^{-\frac{1}{2}} f_{B_{r_{k}}} u_{k}\left(\zeta+\zeta_{k}+z^{j_{k}}\right) \mathrm{d} \xi \mathrm{d} \eta \varphi(z) \mathrm{d} x \mathrm{~d} y \\
& \leq C \sup _{\zeta \in B} \int_{B} j_{k}^{-\frac{1}{2}} u_{k}\left(z^{j_{k}}+\zeta\right) \mathrm{d} x \mathrm{~d} y .
\end{aligned}
$$

With suitable $\zeta_{k}^{\prime} \in B$ one can estimate the last expression by

$$
\left|\int_{B} j_{k}^{-\frac{1}{2}} u_{k}\left(z^{j_{k}}+\zeta_{k}^{\prime}\right) \mathrm{d} x \mathrm{~d} y\right|=\left|\int_{B} g_{j_{k}, \zeta_{k}^{\prime}} u_{k}(z) \mathrm{d} x \mathrm{~d} y\right| .
$$

Since $u_{k} \stackrel{D}{\rightarrow} 0$, the right-hand side converges to zero by Lemma 3.1.

Lemma 3.3. Let $\bar{\Omega} \subset B$. Then there exists $C>0$ such that for every $w \in L^{2}(\Omega)$, extended by zero to $\mathbb{R}^{2}$, and for every small $r>0$,

$$
\left|A_{r} w\left(z^{\prime}\right)\right| \geq\left|A_{r} w(z)\right|-C\|w\|_{2} \frac{\left|z-z^{\prime}\right|^{\frac{1}{2}}}{r^{\frac{3}{2}}}, z, z^{\prime} \in B .
$$

Proof. From the definition of the averaging operator $A_{r}$, by the Cauchy inequality, and denoting the symmetric difference of sets $(A \cup B) \backslash(A \cap B)$ as $A \triangle B$, we have

$$
\begin{aligned}
\left|A_{r} w\left(z^{\prime}\right)-A_{r} w(z)\right| & \leq C r^{-2} \int_{B_{r}\left(z^{\prime}\right) \triangle B_{r}(z)}|w| \mathrm{d} x \mathrm{~d} y \\
& \leq C r^{-2}\|w\|_{2} \sqrt{\left|B_{r}\left(z^{\prime}\right) \triangle B_{r}(z)\right|} \\
& \leq C\|w\|_{2} r^{-2} \sqrt{r\left|z-z^{\prime}\right|}=C\|w\|_{2} \frac{\left|z-z^{\prime}\right|^{\frac{1}{2}}}{r^{\frac{3}{2}}}
\end{aligned}
$$

from which (3.2) is immediate.

In what follows $w^{\star}$ denotes the symmetric decreasing rearrangement of $w$.

Lemma 3.4. Let $u_{k}$ be a bounded sequence in $H_{0}^{1}(\Omega)$. If for every $j_{k} \in \mathbb{N}$ the sequence $g_{j_{k}, 0} u_{k}^{\star}$ converges to zero in measure then $u_{k} \rightarrow 0$ in $\exp L^{2}$.

Proof. Since convergence in measure, for sequences bounded in $H_{0}^{1}(B)$, implies weak convergence in $H_{0}^{1}(B)$, it follows from identity (B.5) that

$$
\int_{B} \nabla m_{e^{-j_{k}}} \cdot \nabla u_{k}^{\star}=\int_{B} \nabla m_{e^{-1}} \cdot \nabla g_{j_{k}, 0} u_{k}^{\star} \rightarrow 0,
$$


The set of isometries $\left\{g_{j, 0}\right\}_{j \in \mathbb{N}}$, once their domain is restricted to the radial subspace $H_{0, r}^{1}(B)$, becomes a subset of the multiplicative group of isometries $\left\{h_{s}\right\}_{s>0}$ defined by (B .4). This group has the following, easily verifiable, property: if $s_{k}$ is a bounded sequence and $v_{k} \rightarrow 0$ in $H_{0, r}^{1}(B)$, then $h_{s_{k}} v_{k} \rightarrow 0$. Then it follows from (3.3) that for any sequence $r_{k} \in\left(0, e^{-1}\right)$ (with $j_{k} \in \mathbb{N}$ chosen so that $0 \leq \log \frac{1}{r_{k}}-j_{k} \leq 1$ ),

$$
\int_{B} \nabla m_{r_{k}} \cdot \nabla u_{k}^{\star} \rightarrow 0 .
$$

Then taking into account (B.1) and (B.2), we conclude that

$$
\sup _{r \in\left(0, e^{-1}\right)} \frac{u_{k}^{\star}(r)}{\left(\log \frac{1}{r}\right)^{\frac{1}{2}}} \rightarrow 0 .
$$

Moreover, by the compactness in the one-dimensional Morrey imbedding, we also have

$$
\sup _{r \in\left[e^{-1}, 1\right)} \frac{u_{k}^{\star}(r)}{\left(1+\log \frac{1}{r}\right)^{\frac{1}{2}}} \rightarrow 0 .
$$

Combining two last relations we arrive at

$$
\sup _{r \in(0,1)} \frac{u_{k}^{\star}(r)}{\left(1+\log \frac{1}{r}\right)^{\frac{1}{2}}} \rightarrow 0,
$$

that is, $u_{k} \rightarrow 0$ in $L^{\infty, 2 ;-1}=\exp L^{2}$.

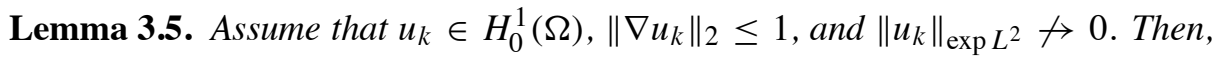
for a renumbered subsequence, there exists a sequence $j_{k} \in \mathbb{N}, \zeta_{k} \in \bar{\Omega}$, such that for every $\epsilon>0$ there exits $\rho>0$ such that

$$
j_{k}^{-\frac{1}{2}}\left|A_{\rho^{j_{k}}} u_{k}\left(\zeta_{k}\right)\right| \geq \epsilon
$$

Proof. Since $u_{k}$ does not converge to zero in $\exp L^{2}$, by Lemma 3.4 there is a renumbered subsequence such that, for any $\epsilon>0$, the measure of the sets

$$
M_{k}^{\star}=\left\{z \in B: j_{k}^{-\frac{1}{2}} u_{k}^{\star}\left(r^{j_{k}}\right) \geq 2 \epsilon\right\}
$$

is bounded away from zero. Since $u^{\star}$ is a decreasing function, there exists $\rho>0$ such that $j_{k}^{-\frac{1}{2}} u_{k}^{\star}(r) \geq 2 \epsilon$ for all $r \leq \rho^{j_{k}}$. This immediately implies that

$$
\left|M_{k}\right| \geq \pi \rho^{2 j_{k}}, \text { where } M_{k}=\left\{z \in \Omega: j_{k}^{-\frac{1}{2}}\left|u_{k}(z)\right| \geq 2 \epsilon\right\}
$$


Let us now use a well-known inequality (see $e . g$. inequality (4) in [16]) that holds for every $u \in H_{0}^{1}(B)$ :

$$
\left\|A_{r} u-u\right\|_{2} \leq C r\|\nabla u\|_{2} .
$$

In particular, we have

$$
\int_{M_{k}}\left|A_{r_{k}} u_{k}-u_{k}\right|^{2} \leq C r_{k}^{2}
$$

which, combined with (3.7), gives

$$
C r_{k}^{2} \geq \pi \rho^{2 j_{k}} \inf _{z \in M_{k}}\left|A_{r_{k}} u_{k}(z)-u_{k}(z)\right|^{2},
$$

from which we conclude that there exists a sequence $\zeta_{k} \in M_{k}$, such that

$$
j_{k}^{-\frac{1}{2}}\left|A_{r_{k}} u_{k}\left(\zeta_{k}\right)\right| \geq 2 \epsilon-C j_{k}^{-\frac{1}{2}} \frac{r_{k}}{\rho^{j_{k}}} \geq \epsilon
$$

from which the assertion of the lemma is immediate once we choose $r_{k}=\rho^{j_{k}}$.

Proof of Theorem 2.6. Assume that there exists a sequence $u_{k} \in H_{0}^{1}(\Omega), u_{k} \stackrel{D}{\rightarrow} 0$, which does not converge to zero in $\exp L^{2}$. Then let $\rho>0, j_{k}$ and $\zeta_{k}$ be as in Lemma 3.5.

Let us fix $\epsilon>0$ and evaluate the measure of the sets

$$
N_{k}=\left\{z \in B:\left|g_{j_{k}, \zeta_{k}} A_{\rho^{j_{k}}} u_{k}(z)\right| \geq \epsilon / 2\right\} .
$$

Applying Lemma 3.3 with $w=j_{k}^{-\frac{1}{2}} u_{k}, r=\rho^{j_{k}}$ and $z^{\prime}=\zeta_{k}$, we have from (3.2) and 3.5, for all $z \in B$ such that $\left|z-\zeta_{k}\right| \leq \rho^{5 j_{k}}$,

$$
j_{k}^{-\frac{1}{2}}\left|A_{\rho^{j_{k}}} u_{k}(z)\right| \geq \epsilon-C \rho^{j_{k}} \geq \epsilon / 2,
$$

for all sufficiently large $k$. Then, from the definition of the set $N_{k}$ above and the definition of $g_{j, \zeta}$ in (2.15), it follows that the set $N_{k}$ contains the ball

$$
\left\{z \in B:|z|^{j_{k}} \leq \rho^{5 j_{k}}\right\}
$$

that is, $N_{k} \supset B_{\rho^{5}}$. 
We conclude that $g_{j_{k}, z_{k}} A_{\rho^{j_{k}}} u_{k}$ does not converge to zero in measure, and thus that $A_{\rho^{j}} u_{k}$ does not converge to zero $D$-weakly. Then, by Lemma 3.2, $u_{k}$ does not converge to zero $D$-weakly, which contradicts the assumption of the theorem.

Proof of Theorem 1.1. Since the Moser functional is lower weakly semicontinuous by the Fatou lemma, and since it is known that it lacks weak continuity on the unit ball of $H_{0}^{1}(B)$ only at zero, we may assume without loss of generality that $u_{k} \in$ $H_{0}^{1}(\Omega)$ is such that $\left\|\nabla u_{k}\right\|_{2} \leq 1, u_{k} \rightarrow 0$ and $\lim J\left(u_{k}\right)>J(0)=0$. Consider a renumbered subsequence of $u_{k}$ satisfying (2.20) and recall that the remainder there converges to zero in $\exp L^{2}$ by Theorem 2.6. Then, leaving details of separating supports to the reader, we have

$$
J\left(u_{k}\right)=\sum_{n \in \mathbb{N}} J\left(v_{k}^{(n)}\right)+o(1) .
$$

where

$$
v_{k}^{(n)}=j_{k}^{(n)^{1 / 2}} w^{(n)}\left(\left|z-z_{n}\right|^{1 / j_{k}^{(n)}}\right)
$$

Note that, since the argument of $J$ converges weakly to zero and $\left\|\nabla v_{k}^{(n)}\right\|_{2}=$ $\left\|\nabla w^{(n)}\right\|_{2}$, one has $J\left(v_{k}^{(n)}\right) \rightarrow 0$ whenever $\left\|\nabla w^{(n)}\right\|_{2}<1$. By assumption, $\lim J\left(u_{k}\right)>0$, which implies that for at least one value of $n,\left\|\nabla w^{(n)}\right\|_{2} \geq 1$. Without loss of generality assume that this value of $n$ is 1 . Comparing this with (2.19), we conclude that $w^{(n)}=0$ whenever $n>1$. Therefore

$$
u_{k}=v_{k}^{(1)}+\omega_{k}
$$

with $\omega_{k} \stackrel{D}{\rightarrow} 0$ and in particular, $\omega_{k}$ vanishes in $\exp L^{2}$.

Note now that, since $\omega_{k} \stackrel{D}{\rightarrow} 0$, one has $\left(\omega_{k}, v_{k}^{(1)}\right) \rightarrow 0$, and, consequently,

$$
1 \geq \lim \sup \left\|\nabla u_{k}\right\|_{2}^{2}=\lim \sup \left\|\nabla v_{k}^{(1)}+\nabla \omega_{k}\right\|_{2}^{2}=1+\lim \sup \left\|\nabla \omega_{k}\right\|_{2}^{2} \text {. }
$$

Therefore $\omega_{k} \rightarrow 0$ in $H^{1}$.

If $w^{(1)}$ is not a Moser function, then by Proposition B.1, $J\left(v_{k}^{(1)}\right) \rightarrow 0$. A slight modification of the proof of Proposition B.1 gives also $J\left(u_{k}\right) \rightarrow 0$, which is a contradiction. Consequently, using (B.5), we have $u_{k}-m_{s_{k}} \rightarrow 0$ in $H^{1}$ with some sequence $s_{k} \rightarrow 0$, which proves the theorem.

\section{A. Optimal imbeddings of Sobolev spaces into Lorentz-Zygmund spaces}

Let $f: \mathbb{R}^{N} \rightarrow \mathbb{R}$ be a measurable function. The distribution function $\alpha_{f}$ and the nonincreasing rearrangement $f^{\star}$ of $f$ are defined as follows:

$$
\alpha_{f}(s)=\left|\left\{x \in \mathbb{R}^{N}:|f(x)|>s\right\}\right| \text { and } f^{*}(t)=\inf \left\{s>0: \alpha_{f}(s) \leq t\right\} .
$$


The Lorentz-Zygmund spaces $L^{p, q ; \alpha}$ constitute a family of spaces that contains both the Lorentz spaces $L^{p, q ; 0}=L^{p, q}$ and the Zygmund spaces $L^{0, \infty ;-\alpha}=Z^{\alpha}$. They are defined as the spaces of all the measurable functions on the unit ball with bounded quasinorms

$$
\begin{aligned}
\|u\|_{p, q ; \alpha} & =\left(\int_{0}^{1}\left[t^{1 / p}\left(\log \frac{e}{t}\right)^{\alpha} f^{\star}(t)\right]^{q} \frac{\mathrm{d} t}{t}\right)^{\frac{1}{q}}, q \in(0, \infty) \\
\|u\|_{p, \infty ; \alpha} & =\sup _{t \in(0,1)}\left|t^{1 / p}\left(\log \frac{e}{t}\right)^{\alpha} f^{\star}(t)\right|, q=\infty .
\end{aligned}
$$

For the purpose of this paper we consider the range $p \in(1, \infty)$. The definition of the Lorentz space $L^{p, q}\left(\mathbb{R}^{N}\right)$ is the same as the definition of $L^{p, q ; 0}$ above with the domain of integration $t \in(0, \infty)$ instead of $t \in(0,1)$.

The Lorentz space $L^{r, r}$ is equivalent to the Lebesgue space $L^{r}$, and the Lorentz space $L^{r, \infty}$ is equivalent to the Marcinkiewicz space $M^{r}$, also known as the weak$L^{r}$ space. The Lorentz-Zygmund space $L^{\infty, \infty,-1 / N^{\prime}}$ is equivalent to the Orlicz space $\exp L^{N^{\prime}}$ of the Moser functional.

For more background material on Lorentz and Lorentz-Zygmund spaces we address the reader to Bennett and Sharpley [6] and Bennett and Rudnick [5].

The reason why the domain of the functions considered here is the unit ball, rather than $\mathbb{R}^{N}$, lies in the role of Lorentz-Zygmund spaces in the imbeddings of Sobolev spaces with the gradient norm. The completion $\mathcal{D}^{1, N}\left(\mathbb{R}^{N}\right)$ of the normed space $C_{0}^{\infty}\left(\mathbb{R}^{N}\right)$ equipped with the norm $\|\nabla u\|_{N}$ does not admit a continuous imbedding even into the space of distributions, which means that the space $\mathcal{D}^{1, N}\left(\mathbb{R}^{N}\right)$ cannot be consistently defined as a function space. On the other hand, Friedrichs' inequality gives that the completion of $C_{0}^{\infty}(B)$ in the same gradient norm $\|\nabla u\|_{N}$ is continuously imbedded into $L^{N}(B)$, defining the space $W^{1, N}(B)$ with the equivalent Sobolev norm $\|\nabla u\|_{N}$. It should be noted as well that $\|\nabla u\|_{N}$ also expresses the gradient norm of the Laplace-Beltrami operator on the hyperbolic space $\mathbb{H}^{N}$ when written under the coordinate map of the Poincare ball, which allows to understand that the "Euclidean" Sobolev space $W_{0}^{1, N}(B)$ of the unit ball in $\mathbb{R}^{N}$ is isometric to the Sobolev space $\dot{W}^{1, N}\left(\mathbb{H}^{N}\right)$ of a complete non-compact Riemannian manifold, giving the unit ball in $\mathbb{R}^{N}$ when $p=N$ an intuitively equal standing, when Sobolev spaces are concerned, with the whole $\mathbb{R}^{N}$ when $p<N$.

In fact, the similarities between $\mathcal{D}^{1, p}\left(\mathbb{R}^{N}\right)$ for $N>p$, and $W_{0}^{1, N}(B)$, equipped with the gradient norm, are quite extensive. In particular, while the norm $\mathcal{D}^{1, p}\left(\mathbb{R}^{N}\right)$ remains invariant under dilations $u \mapsto t^{\frac{N-p}{p}} u(t \cdot)$ also in the case $p=N$ (even if $\mathcal{D}^{1, N}\left(\mathbb{R}^{N}\right)$ is no longer a functional space), the subspace of radial functions of $W_{0}^{1, N}(B)$ admits a different isometry group (B.4) of nonlinear dilations. There are also similarities in imbeddings of Sobolev type into rearrangement-invariant spaces. While the standard limiting Sobolev inequality and the Trudinger-Moser inequality are quite different in appearance, this difference finds its explanation when one 
considers imbeddings of Sobolev spaces into the scales of correspondent Lorentz or Lorentz-Zygmund spaces.

We observe first that there is a continuous imbedding of $\mathcal{D}^{1, p}\left(\mathbb{R}^{N}\right), N>p$, into $L^{p^{*}, p}$, which is immediate from the Hardy inequality for $u^{\star}$, combined with the Polia-Szegö inequality. The analogous imbedding for $p=N$ is $W_{0}^{1, N}(B) \hookrightarrow$ $L^{\infty, N ;-1}$, based on the inequality of Hardy type

$$
\int_{B}|\nabla u|^{N} \geq C_{N} \int_{B} \frac{|u|^{N}}{\left(r \log \frac{1}{r}\right)^{N}}, u \in C_{0}^{\infty}(B) .
$$

(see Adimurthi and Sandeep [3] and Adimurthi and Sekar [2]; in the case $N=2$ this was proved first by Leray [11]).

Lorentz spaces are nested with respect to the second index, and thus there is a continuous imbedding $\mathcal{D}^{1, p}\left(\mathbb{R}^{N}\right) \hookrightarrow L^{p^{*}, q}, N>p$, for all $q \in[p, \infty]$

A continuous imbedding $W_{0}^{1, N}(B) \hookrightarrow L^{\infty, \infty ;-1 / N^{\prime}}$ follows from the inequality (B.6) for radial functions (see e.g. [13]) for $p=N$, combined with the PoliaSzegö inequality, and the Hölder inequality yields therefore the following family of imbedding into Lorentz-Zygmund spaces when $p=N: W_{0}^{1, N}(B) \hookrightarrow L^{\infty, q ;-1 / q-1 / N^{\prime}}$ for $p \leq q \leq \infty$.

The smallest of the target spaces corresponds to $q=p$ in both cases, and, moreover, these imbeddings are optimal in the class of rearrangement-invariant spaces (see [6] for the definition), shown by Peetre [14] in the case $N>p$ and by Brezis and Wainger [7] in the case $p=N$.

The limiting Sobolev inequality and the Trudinger-Moser inequality are optimal in the sense that one cannot replace the correspondent nonlinearity by any other with a faster growth. Indeed, if $h(s)$ is any continuous non-decreasing unbounded function on $[0, \infty)$, then

$$
\sup _{u \in W_{0}^{1, N}(B),\|\nabla u\|_{N} \leq 1} \int_{B} h(|u|) e^{\alpha_{N}|u|^{N^{\prime}}} \mathrm{d} x=+\infty,
$$

and

$$
\sup _{u \in \mathcal{D}^{1, p}\left(\mathbb{R}^{N}\right),\|\nabla u\|_{p} \leq 1} \int_{\Omega} h(|u|)|u|^{p^{*}} \mathrm{~d} x=+\infty .
$$

In the case $N>p$ this can be immediately seen by evaluating the functional on $t^{\frac{N-2}{2}} u_{0}(t x)$ with $t>0$ and $u_{0} \neq 0$. In the case $p=N$, one arrives at the similar conclusion by evaluation of the functional on the Moser family of functions (1.3) normalized in the norm of $W_{0}^{1, N}(B)$.

\section{B. The Moser functional in the radial case}

In this appendix we list some basic properties of the Moser functional on the radial subspace $W_{0, r}^{1, N}(B)$ of $W_{0}^{1, N}(B)$. In particular we show that, restricted to radial 
functions, it is weakly continuous on any sequence that is not, asymptotically, a sequence of concentrating Moser functions. This conclusion can be inferred from the original paper by Moser [13], while the notation we use here are brought from the paper [4]. The calculations involved in this proof also allow to present the original Moser's proof of the Trudinger-Moser inequality in a concise and streamlined form.

Let $m_{t}$, with $t \in(0,1)$, be the family of Moser functions (1.3), and consider the following functional on $W_{0, r}^{1, N}(B)$ :

$$
\left\langle m_{t}^{*}, u\right\rangle=\int_{B}\left|\nabla m_{t}\right|^{N-2} \nabla m_{t} \cdot \nabla u \mathrm{~d} x, \quad t \in(0,1) .
$$

An elementary computation shows that the functional $m_{t}^{*}$ is continuous and

$$
\left\langle m_{t}^{*}, u\right\rangle=\omega_{N-1}^{1 / N} \log (1 / t)^{-1 / N^{\prime}} u(t), \quad t \in(0,1) .
$$

Proposition B.1. Let $u_{k} \in W_{0, r}^{1, N}(B),\left\|\nabla u_{k}\right\|_{N} \leq 1, u_{k} \rightarrow u$ and let $J$ be the Moser functional (1.2). Then $J\left(u_{k}\right) \rightarrow J(u)$, unless the sequence $u_{k}$ has a renamed subsequence such that $u_{k}-m_{t_{k}} \rightarrow 0$ in $W_{0, r}^{1, N}(B)$, with $t_{k} \rightarrow 0$.

Proof. Let us substitute (B.2) into the definition of the Moser functional. After elementary simplifications one arrives to the following representation

$$
J(u)=\omega_{N-1}\left(\int_{0}^{1} r^{N\left(1-\left\langle m_{t}^{*}, u\right\rangle^{N}\right)} \frac{\mathrm{d} r}{r}-1 / N\right),
$$

where $u \in W_{0, r}^{1, N}(B)$ and $\|\nabla u\|_{N}=1$. Assume first that there exists $\epsilon>0$ such that $\left\langle m_{t}^{*}, u_{k}\right\rangle^{N} \leq 1-\epsilon$. Then $J\left(u_{k}\right) \rightarrow J(u)$ by the Lebesgue dominated convergence theorem. The remaining case is when for some $t_{k} \in(0,1), u_{k}-m_{t_{k}} \rightarrow 0$ in $W_{0, r}^{1, N}(B)$. Assume first that the weak limit $u$ is not zero. Then, necessarily, $u_{k} \rightarrow$ $m_{t}$ in $W_{0, r}^{1, N}(B)$ for some $t \in(0,1)$. This implies the uniform convergence of $u_{k}$ on $[t, 1]$ as well as $\int_{B_{t}}\left|\nabla u_{k}\right|^{N} \mathrm{~d} x \rightarrow 0$, from which easily follows $J\left(u_{k}\right) \rightarrow J\left(m_{t}\right)$. If $u_{k}=m_{t_{k}}+o(1) \rightarrow 0$ with $t_{k} \rightarrow 1$, an argument repetitive of that for the case $u_{k} \rightarrow m_{t}$ above will give $J\left(u_{k}\right) \rightarrow 0=J(u)$. We have, therefore, with necessity, a renamed subsequence $u_{k}=m_{t_{k}}+o(1)$ with $t_{k} \rightarrow 0$.

Let

$$
h_{s} u(r) \stackrel{\mathrm{d} e f}{=} s^{-1 / N^{\prime}} u\left(r^{s}\right), s>0, u \in W_{0, r}^{1, N}(B),
$$

Elementary calculations show that the operators (B.4) form a multiplicative group of linear isometries on $W_{0, r}^{1, N}(B)$. Furthermore, for every $s>0$ and $t \in(0,1)$,

$$
h_{s} m_{t}=m_{t^{1 / s}} .
$$

We also note a well-known radial estimate: for each $u \in W_{0, r}^{1, N}(B)$,

$$
\sup _{r \in(0,1)}|u(r)|\left(\log (1 / r)^{-1 / N^{\prime}} \leq \omega_{N-1}^{-1 / N}\|\nabla u\|_{N} .\right.
$$




\section{References}

[1] D. R. ADAMS, A sharp inequality of J. Moser for higher order derivatives, Ann. of Math. (2) 128 (1988), 385-398.

[2] Adimurthi and A. Sekar, Role of the fundamental solution in Hardy-Sobolev-type inequalities, Proc. Roy. Soc. Edinburgh Sect. A 136 (2006), 1111-1130.

[3] Adimurthi and K. SANDEEP, Existence and non-existence of the first eigenvalue of the perturbed Hardy-Sobolev operator, Proc. Roy. Soc. Edinburgh Sect. A 132 (2002), 10211043.

[4] Adimurthi, J. M. Do Ó and K. TinTAREV, Cocompactness and minimizers for inequalities of Hardy-Sobolev type involving $N$-Laplacian, NoDEA Nonlinear Differential Equations Appl. 17 (2010) 467-477.

[5] C. Bennett and K.Rudnick, On Lorentz-Zygmund spaces, Dissertationes Math. 175 (1980) 1-72.

[6] C. BennetT and R. Sharpley, "Interpolation of Operators", Pure and Applied Mathematics, Vol. 129, Academic Press, Boston, 1988.

[7] H. BreZIS and S. WAINGER, A note on limiting cases of Sobolev embeddings and convolution inequalities, Comm. Partial Differential Equations 5 (1980), 773-789.

[8] N. Fusco, P.-L. Lions and C. Sbordone, Sobolev imbedding theorems in borderline cases, Proc. Amer. Math. Soc. 124 (1996), 561-565.

[9] P. GÉRARD, Description du dèfaut de compacité de l'injection de Sobolev, ESAIM Control Optim. Calc. Var. 3 (1998), 213-233.

[10] S. JAFFARD, Analysis of the lack of compactness in the critical Sobolev embeddings, J. Funct. Anal. 161 (1999), 384-396.

[11] J. LERAY, Sur le mouvement d'un liquide visqueux emplissant l'espace, Acta Math. 63 (1934), 193-248.

[12] P.-L. Lions, The concentration-compactness principle in the calculus of variations. The limit case II, Rev. Mat. Iberoam. 1 (1985), 45-121.

[13] J. Moser, A sharp form of an inequality by N. Trudinger, Indiana Univ. Math. J. 20 (1971) 1077-1092.

[14] J. PeETRE, Espaces d'interpolation et thórème de Soboleff, Ann. Inst. Fourier (Grenoble) 16 (1966), 279-317.

[15] S. I. PohozhaeV, The Sobolev imbedding in the case $p l=n$, In: "Proc. Tech. Sci. Conf. on Adv. Sci. Research 1964-1965", Mathematics Section, Moskov. Energet. Inst., Moscow 1965, 158-170.

[16] S. Solimini, A note on compactness-type properties with respect to Lorentz norms of bounded subsets of a Sobolev space, Ann. Inst. H. Poincaré Anal. Non Linéaire 12 (1995), 319-337.

[17] W. A. STRAuSs, Existence of solitary waves in higher dimensions, Comm. Math. Phys. 55 (1977), 149-162.

[18] M. STRUWE, A global compactness result for elliptic boundary value problems involving limiting nonlinearities, Math. Z. 187 (1984), 511-517.

[19] I. SCHINDLER and K. TINTAREV, An abstract version of the concentration compactness principle, Rev. Mat. Complut. 15 (2002), 417-436.

[20] K. Tintarev and K.-H. Fieseler, "Concentration Compactness: Functional-analytic Grounds and Applications", Imperial College Press, London, 2007.

[21] N. S. TRUdinger, On imbeddings into Orlicz spaces and some applications, J. Math. Mech. 17 (1967), 473-483. 
[22] V. I. Yudovich, Some estimates connected with integral operators and with solutions of elliptic equations, Sov. Math. Dokl. 2 (1961), 746-749.

\section{TIFR CAM}

Sharadanagar, P.B. 6503

Bangalore 560065, India aditi@math.tifrbng.res.in

Uppsala University

P.O.Box 480

SE-751 06 Uppsala, Sweden tintarev@math.uu.se 